\title{
Estado nutricional según IMC en estudiantes de cuatro Escuelas Académicas Profesionales de la Facultad de Medicina UNMSM
}

\author{
María del Pilar Cereceda, Sissy Espinoza, Dina Apaza
}

Facultad de Medicina UNMSM

\begin{abstract}
Introducción: En nuestro país, existen pocos estudios en estudiantes universitarios que nos permita tener información necesaria para orientar adecuadamente algunas acciones de intervención. En relación a esto, surgió la necesidad de investigar el estado nutricional de los estudiantes de la Facultad de Medicina.

Objetivos: Determinar el estado nutricional, según IMC, de estudiantes de 4 Escuelas de la Facultad de Medicina.

Diseño: Estudio descriptivo de corte transversal.

Institución: Facultad de Medicina, UNMSM.

Participantes: Estudiantes de las Escuelas de Obstetricia, Nutrición, Enfermería y Tecnología Médica de la Facultad de Medicina.

Intervenciones: La muestra fue seleccionada por conveniencia y estuvo integrada por 94 estudiantes de ambos sexos de cuatro Escuelas de la Facultad de Medicina (Obstetricia, Nutrición, Enfermería y Tecnología Médica). Se realizó mediciones de peso y talla y luego se determinó el estado nutricional según IMC, considerando los puntos de corte recomendado por la OMS. Se contó con la participación de estudiantes de la EAP de Nutrición y egresados, que ayudaron con la toma de datos y procesamiento de la información.

Principales medidas de resultados: Presencia de sobrepeso en alumnos de las 4 EAPs evaluadas.

Resultados: Se encontró una prevalencia de sobrepeso de $14,9 \%$, obesidad de $3,2 \%$ y 5,3 de delgadez, siendo la prevalencia de sobrepeso y obesidad similar en las cuatro Escuelas.

Conclusiones: Se encontró sobrepeso, obesidad y delgadez en la muestra de estudio.

Palabras clave: Sobrepeso, indice de masa corporal, estado nutricional.
\end{abstract}

\section{Perfil clínico-epidemiológico de pacientes con estrongiloidiasis atendidos en el Instituto de Medicina Tropical D. A. Carrión 2005-2011}

\author{
Herman Vildózola, Miguel Gusukuma, Yrma Espinoza
}

Instituto de Medicina Tropical, Facultad de Medicina, UNMSM

Objetivos: Definir el perfil clínico-epidemiológico de pacientes con estrongiloidiasis atendidos en el Instituto de Medicina Tropical, entre los años 2005 y 2011.

Diseño: Estudio prospectivo de serie de casos.

Institución: Instituto de Medicina Tropical, UNMSM.

Participantes: Pacientes con diagnóstico de estrongiloidiasis.

Intervenciones: A 31 pacientes con diagnóstico de estrongiloidiasis, 20 varones y 11 mujeres, se les hizo ficha clínicoepidemiológica, examen de heces seriado con método de Baerman y sedimentación rápida, hemograma completo.

Principales medidas de resultados: Porcentajes de síntomas y signos, procedencia, vivir o haber estado en la selva y recuento de eosinófilos.

Resultados: La edad promedio de los 31 pacientes fue 40 años, $41,9 \%$ nació en la selva, $32,2 \%$ en la costa y $25,8 \%$ en la sierra; $25,8 \%$ vivia en la selva, $61,2 \%$ vivía en Lima, pero en algún momento vivió en la selva y $13 \%$ nunca estuvo en la selva. Tiempo de enfermedad entre 1 mes y 25 años, diarrea crónica $54,8 \%$, dolor abdominal $51,6 \%$, náuseas $35,4 \%$, gases, vómitos y ruidos intestinales en $25,8 \%, 45,1 \%$ había perdido peso entre 2 y $17 \mathrm{~kg}, 45,1 \%$ tenía eosinofilia mayor a 400 .

Conclusiones: Epidemiológicamente, el factor más importante fue vivir o haber vivido en la selva; los síntomas predominantes fueron diarrea crónica, dolor abdominal, náuseas y eosinofilia, en la mitad de los pacientes.

Palabras clave: Estrongiloidiasis, sintomas, epidemiología, eosinofilia. 